\title{
Heterogeneity in responses by primary adult human colonic epithelial cells to purified enterotoxin of Bacteroides fragilis
}

\author{
L Sanfilippo, T J Baldwin, M G Menozzi, S P Borriello, Y R Mahida
}

\begin{abstract}
Background-Enterotoxigenic strains of Bacteroides fragilis (ETBF) have been implicated in diarrhoeal illness in livestock and children, but their role in adult human colonic disease is unknown.

Aims-To investigate responses by primary adult human colonic epithelial cells to purified $B$ fragilis toxin (BFT).

Methods-BFT was purified from culture supernatant of a highly toxigenic strain of ETBF. Morphological changes to primary colonic epithelial cells, in response to purified BFT, were studied in organ culture of colonic biopsy specimens from 15 adults.

Results-BFT induced epithelial cell cytotoxicity in colonic biopsy specimens from $12 / 15$ subjects. The BFT induced morphological changes were characterised by epithelial cell rounding, separation from adjacent cells, and detachment from the basement membrane. In severely affected specimens, almost all the epithelial cells were affected. There was heterogeneity between subjects in the rate at which BFT induced epithelial cell cytotoxicity occurred. Furthermore, in colonic biopsy specimens from three subjects, exposure to BFT did not induce any significant morphological changes to epithelial cells. Conclusion-BFT is capable of inducing cytotoxicity in primary adult human colonic epithelial cells. Such an effect of ETBF derived BFT on epithelial cells in the colon in vivo would be expected to lead to mucosal inflammation and diarrhoea. Heterogeneity in responses by primary colonocytes probably reflects the outcome of host-BFT interactions. Such interactions in vivo could determine the occurrence of colonic disease in some individuals but not others.

(Gut 1998;43:651-655)
\end{abstract}

Keywords: Bacteroides fragilis; enterotoxin; epithelial cells; apoptosis

Bacteroides fragilis is a Gram negative bacillus which is normally resident in the colonic lumen of humans and animals. ${ }^{2}$ In the 1980 s, enterotoxin producing strains of $B$ fragilis (ETBF) were isolated from the faeces of young farm animals. ${ }^{3-6}$ Enterotoxigenic activity of these strains was shown by their ability to elicit fluid accumulation in lamb ligated intestinal loops. ${ }^{3}$ In subsequent studies, the enterotoxin was shown to induce rounding of cells of the human carcinoma cell line HT29 and based on this characteristic, an in vitro assay for the detection of ETBF has been developed. ${ }^{7-9}$ The ETBF enterotoxin has recently been purified and shown to be a $20 \mathrm{kDa}$ protein with metalloprotease activity. ${ }^{10} 11$ When injected into intestinal loops, purified $B$ fragilis toxin (BFT) induces fluid accumulation and inflammation ${ }^{12}$ and induces a cytotoxic response on HT29 cells. ${ }^{10}$

In three studies, ETBF has been associated with diarrhoeal illness in children over one year old. ${ }^{13-15}$ Although ETBF has been isolated from stool samples of adult humans, ${ }^{16}{ }^{17}$ its capacity to induce intestinal disease in these individuals is unknown. As ETBF has been detected in stool samples of normal adults as well as those with diarrhoea, it is possible that the bacterium may be able to induce colonic disease, via secreted BFT, in some individuals but not in others. To cause disease, the first host cells that BFT would have to interact with in the colon are epithelial cells. We have investigated epithelial cell responses to BFT in organ cultures of colonic biopsy specimens obtained from adults undergoing colonoscopy.

\section{Materials and methods}

PURIFICATION OF BFT

BFT was purified from a highly toxigenic strain of ETBF (NCTC 11295) as previously described by Van Tassel et al. ${ }^{10}$ Briefly, $B$ fragilis NCTC 11295 was grown anaerobically at $37^{\circ} \mathrm{C}$ in prereduced brain heart infusion broth for 16-18 hours. BFT was purified from culture supernatant by sequential ammonium sulphate precipitation, ion exchange chromatography on Q-Sepharose (Pharmacia Biotech, Brussels, Belgium), hydrophobic interaction chromatography on phenyl agarose (Sigma Chemical Co. Ltd, St Louis, Missouri, USA), and high resolution ion exchange chromatography on a Mono-Q column (Pharmacia Biotech, Brussels, Belgium).

Purification of the toxin was monitored by its cytotoxic effect on HT29 cells (obtained from European Collection of Animal Cell Cultures, Porton Down, UK) characterised by cell rounding. ${ }^{7-9}$ The cytotoxic titre of BFT was expressed as the reciprocal of the highest dilution of the toxin that causes rounding of more than $50 \%$ of HT29 cells after four hours. ${ }^{818} \mathrm{~A}$ cytotoxic unit (CU) was defined as the lowest amount of the toxin that elicited a positive response in HT29 cells (50\% cell rounding at four hours). The cytotoxic titre of our purified 
(stock) BFT ranged from 10240 to 40960 CU, with protein concentration of $1-5 \mu \mathrm{g} / \mathrm{ml}$. BFT was frozen $\left(-20^{\circ} \mathrm{C}\right)$ in aliquots immediately after purification and its titre was retested before and after application to colonic biopsy specimens in organ culture.

Neutralisation of the cytotoxicity of the purified BFT was studied using goat anti-BFT antiserum (gift from Dr TD Wilkins, Virginia Polytechnic Institute and State University, Blacksburg, Virginia, USA). ${ }^{19}$

ORGAN CULTURE STUDIES

Colonic biopsy specimens were obtained from patients undergoing colonoscopy for clinical indications (surveillance for polyps, investigation for occult bleeding, and change in bowel habit). In addition to biopsy specimens for routine examination (which were all confirmed to be normal), additional samples were taken from the sigmoid colon for organ culture studies. These studies were approved by the ethics committee of Queen's Medical Centre and the additional samples were only obtained after informed consent.

Organ culture studies were performed in pairs using separate biopsy specimens obtained from the same area of the sigmoid colon. Organ culture was carried out as previously described, ${ }^{20}$ except that the specimens were cultured in the absence of serum (in RPMI only). In brief, colonic biopsy specimens were placed immediately in RPMI (Gibco BRL, Gaithersburg, Maryland, USA), and within five minutes of removal, placed on a stainless steel mesh over a culture dish (Falcon, Becton Dickinson, Lincoln, New Jersey, USA) containing prewarmed $\left(37^{\circ} \mathrm{C}\right) \mathrm{RPMI}$ and $\mathrm{BFT}$ (at final concentration of $256-2048 \mathrm{CU} / \mathrm{ml}$ ) or the same volume of control buffer. The culture dishes were placed in a sealed chamber, equilibrated with $95 \% \mathrm{O}_{2} / 5 \% \mathrm{CO}_{2}$, and incubated at $37^{\circ} \mathrm{C}$. After varying time intervals (two, four, and 18 hours), biopsy specimens were placed in either $0.9 \% \mathrm{NaCl}$ containing $10 \%$ formalin (for routine histological processing) or in fixative for electron microscopy. Samples were processed for electron microscopy as described previously. $^{21}$

\section{Results}

PURIFICATION OF BFT

Purification of BFT was confirmed by sodium dodecyl sulphate polyacrylamide gel electrophoresis (SDS-PAGE) analysis followed by silver staining, which showed the presence of a single band of protein of $20 \mathrm{kDa}$. Immunoreactivity of purified BFT was also confirmed by western blot analysis (not shown). Cytotoxicity of BFT on HT29 cells was abolished by preincubation with anti-BFT neutralising antibody (data not shown).

\section{ORGAN CULTURE STUDIES}

Morphological changes to epithelial cells were studied by light and transmission electron microscopy of tissue sections of colonic biopsy specimens cultured in the presence or absence of BFT. The effect of BFT was only studied when there was preservation of epithelial integrity in the paired control specimen. In organ culture studies of specimens from a total of 20 subjects, two were excluded from analysis because sections of the control specimens were unsatisfactory.

In preliminary studies, exposure of colonic biopsy specimens from three adult subjects to unfractionated supernatant of ETBF (16-32 $\mathrm{CU} / \mathrm{ml}$ ) did not cause any morphological changes to epithelial cells. All subsequent organ culture studies were performed using purified BFT.

Exposure to purified BFT (256-2048 CU/ $\mathrm{ml}$ ) induced epithelial cell cytotoxicity in specimens from $12 / 15$ subjects (table 1 ). In general, the sequence of changes in the affected specimens was characterised by initial focal areas of epithelial cell rounding, separation from neighbouring cells, and detachment from the basement membrane (fig 1A). These early changes were often initially confined to the surface epithelial cells (fig 1A) with subsequent involvement of crypt epithelial cells after prolonged culture. In the severely affected specimens, the majority of cells were detached from the basement membrane (fig 1B). In addition to vacuolation, a number of the detached, separated colonocytes showed mor-

Table 1 Responses to Bacteroides fragilis toxin (BFT) by epithelial cells in organ cultures of colonic biopsy samples from different subjects

\begin{tabular}{|c|c|c|c|c|c|c|}
\hline \multirow[b]{2}{*}{ Subject } & \multirow[b]{2}{*}{ Age (years) } & \multirow[b]{2}{*}{ Sex } & \multirow[b]{2}{*}{ BFT applied (CUs) } & \multicolumn{3}{|c|}{ Duration of exposure (hours) } \\
\hline & & & & 2 & 4 & 18 \\
\hline 1 & 81 & M & 256 & $\mathrm{NC}$ & C (patchy surface) & NT \\
\hline 2 & 75 & $\mathrm{~F}$ & 512 & C (patchy surface) & C (surface) & NT \\
\hline 3 & 40 & $M$ & 2048 & C (patchy surface) & NT & C (surface and crypt) \\
\hline 4 & 32 & $\mathrm{~F}$ & 2048 & C (patchy surface) & C (surface and crypt) & NT \\
\hline 5 & 61 & $\mathrm{~F}$ & 2048 & C (patchy surface) & NT & C (surface and crypt) \\
\hline 6 & 60 & M & 2048 & C (patchy surface) & C (surface and crypt) & NT \\
\hline 7 & 83 & M & 2048 & NT & C (surface and crypt) & NT \\
\hline 8 & 50 & $\mathrm{~F}$ & 2048 & NT & NC & C (surface and crypt) \\
\hline 9 & 52 & $\mathrm{~F}$ & 2048 & NT & C (surface and crypt) & NT \\
\hline 10 & 53 & $\mathrm{~F}$ & 2048 & NT & C (patchy surface) & C (surface and crypt) \\
\hline 11 & 32 & $\mathrm{~F}$ & 2048 & NT & C (patchy surface) & C (surface and crypt) \\
\hline 12 & 26 & $\mathrm{M}$ & 2048 & NT & C (patchy surface) & C (surface and crypt) \\
\hline 13 & 59 & $\mathrm{~F}$ & 512 & NC & NT & NC \\
\hline 14 & 50 & $\mathrm{~F}$ & 2048 & NT & NC & NT \\
\hline 15 & 77 & $\mathrm{M}$ & 2048 & NT & NC & NT \\
\hline
\end{tabular}

Responses by epithelial cells were characterised, sequentially over time, by involvement of focal areas of surface epithelium ("patchy surface"), whole (or most) of the surface epithelium ("surface"), and crypt epithelial cells ("crypt"). The final concentration of BFT protein in the the organ cultures ranged from 100 to $300 \mathrm{ng} / \mathrm{ml}$.

$\mathrm{C}$, cytotoxicity observed; NC, no cytotoxicity observed; NT, not tested. 

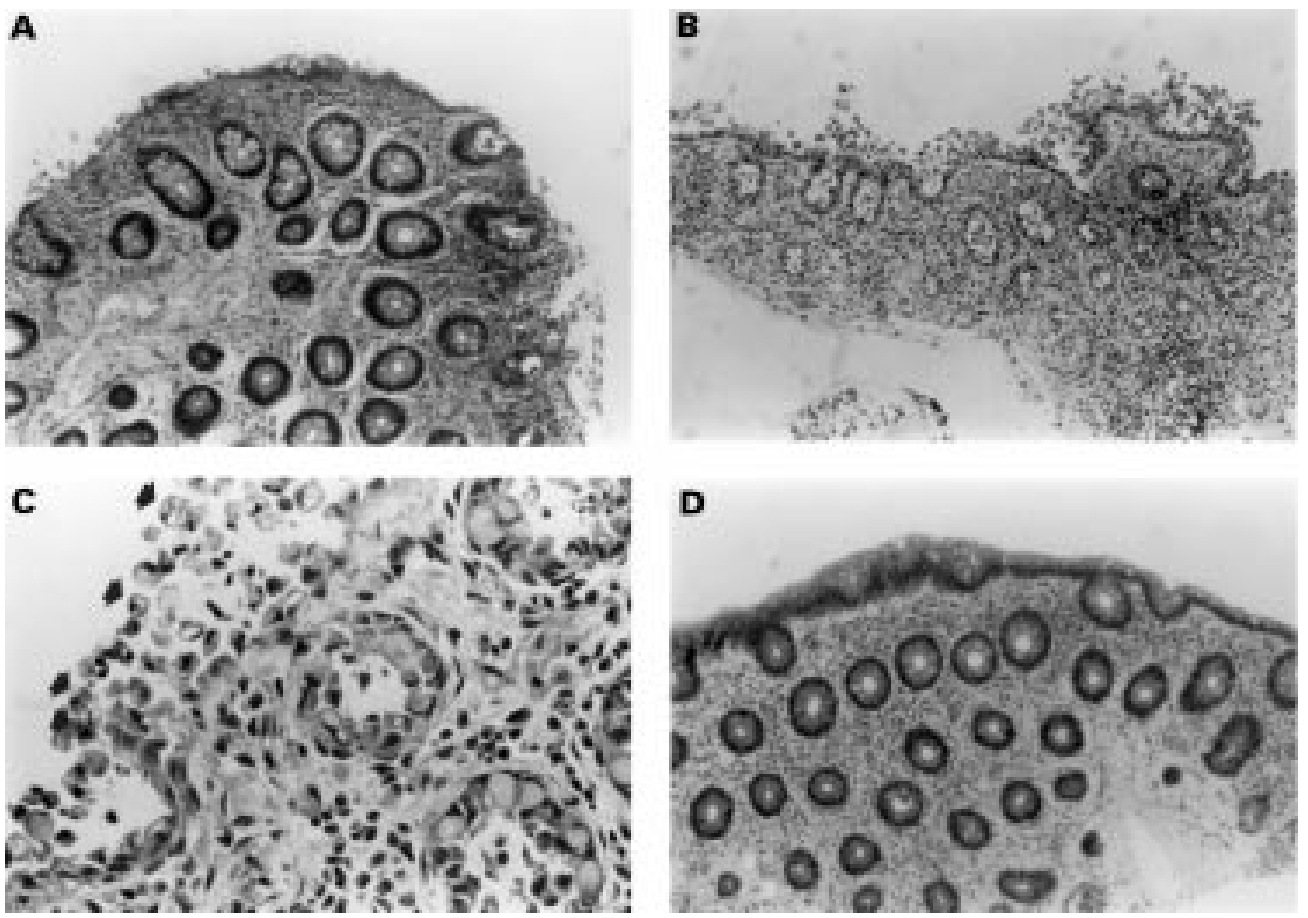

Figure 1 Photomicrographs of haematoxylin and eosin stained sections of colonic biopsy specimens (subject 3 in table 1) exposed to BFT for two hours $(A)$ and 18 hours $(B, C)$. BFT was used at a final concentration of 2048 CU/ml. The paired control specimen (D) was cultured in RPMI (+ control buffer) for 18 hours. There is preservation of epithelial integrity in the control biopsy sample. In the specimen exposed to the toxin for two hours there is focal detachment of surface epithelial cells (patchy effect). After 18 hours exposure to BFT both the surface and crypt epithelial cells are severely affected: they are rounded and most have become detached from the basement membrane and from each other. As shown in (C), there is epithelial cell rounding, detachment from the basement membrane, and vacuolation. Some cells show morphological features of apoptosis (arrowed).

phological features of cells undergoing apoptosis (figs $1 \mathrm{C}, 2 \mathrm{~B}, \mathrm{C}$ ).

There was heterogeneity between subjects in the rate at which BFT induced epithelial cell changes occurred. Thus in one subject (no 8 in table 1), no epithelial cell changes were seen at four hours but mild to moderate BFT induced epithelial cell cytotoxicity was seen after culture for 18 hours. Colonic biopsy specimens from this subject were exposed to the highest concentration of BFT $(2048 \mathrm{CU} / \mathrm{ml})$ used in this study. In another subject (no 1 in table 1), no changes were seen after culture with BFT for two hours, but focal epithelial cytotoxicity was observed after four hours. In colonic biopsy specimens from three subjects (nos 13-15 in table 1), exposure to BFT (for 2-18 hours) did not induce any significant morphological changes in the epithelial cells. Cytotoxic activity of BFT was reconfirmed at the end of all organ culture studies, by applying the culture supernatant to HT29 cells.

\section{Discussion}

Although ETBF has been associated with diarrhoeal illness in children, ${ }^{13-15}$ its role in causing intestinal disease in adults is unknown. BFT has been detected in stool samples of adults with diarrhoea, with titres ranging from 80 to 5120 CU. ${ }^{17}$ Thus, the enterotoxin is present in the colonic lumen in vivo in concentrations used in this study. We believe that unfractionated supernatant of ETBF did not induce any morphological changes to the epithelial cells because only low concentrations of BFT (16-32 CU) were present. Our study shows that in the presence of high concentrations of $\mathrm{BFT}$, cytotoxicity is seen in primary human colonic epithelial cells of adults. The BFT induced morphological changes to the colonic epithelial cells are similar to those described in the intestinal tissue of animals naturally infected with $\mathrm{ETBF}^{22}$ or following intraluminal inoculation of either ETBF or purified BFT. $^{12}{ }^{23-25}$ These changes include areas of focal involvement of the epithelium with cell rounding, vacuolation, separation from neighbouring cells, and detachment from the basement membrane. BFT induced morphological changes in our study are also similar to those observed following exposure of primary human colonocytes to purified Clostridium difficile toxin A. ${ }^{20} C$ difficile is the aetiological agent of antibiotic associated pseudomembranous colitis and the disease is mediated via secreted toxins. ${ }^{26} \mathrm{C}$ difficile toxin A also induces epithelial cell production of the potent polymorphonuclear cell chemoattractant, interleukin 8 (IL-8), ${ }^{20}$ which may play an important role in initiating colonic inflammation in humans. ${ }^{28}$ Physical detachment of primary human colonic epithelial cells, from each other and from the basement membrane, also induces IL- 8 production and release (and subsequent colonocyte death by apoptosis). ${ }^{20}$ As shown in our present study, and reported in a recent preliminary communication, ${ }^{29}$ BFT also induces detachment of primary human colonic epithelial cells. We therefore postulate that BFT would be capable of initiating colonic inflammation in vivo by injuring colonocytes and inducing IL-8 release. Indeed, injection of 


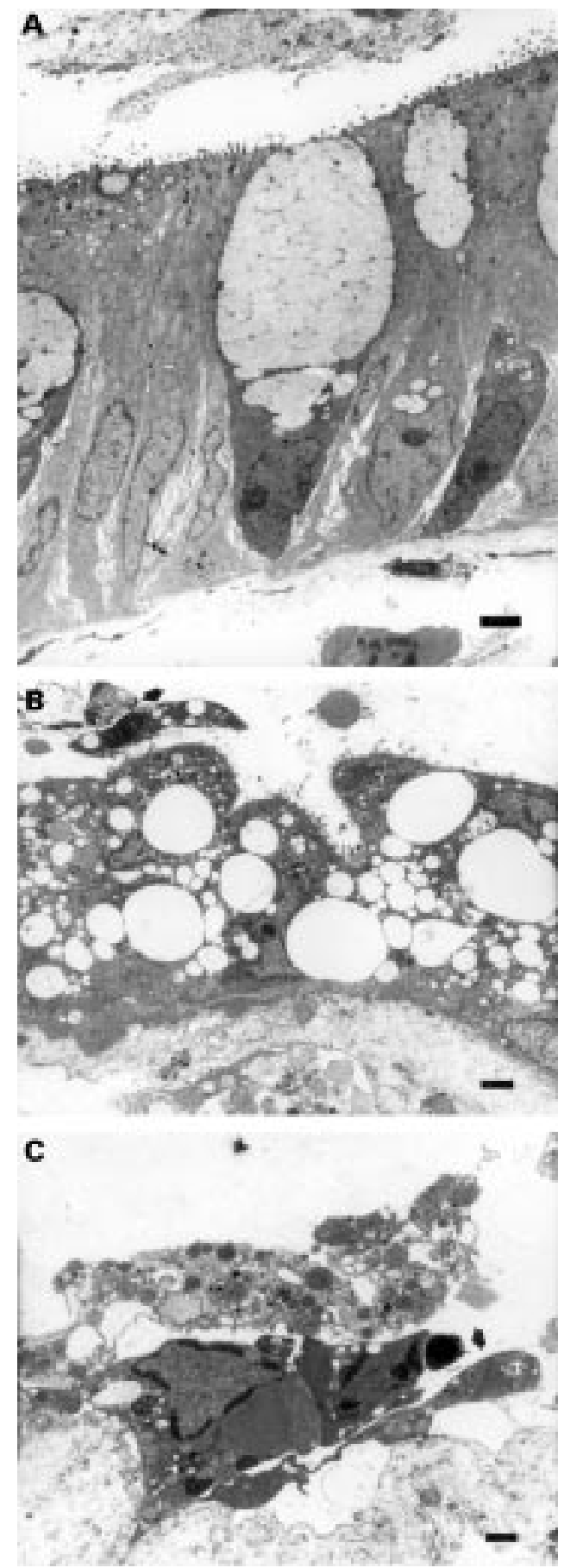

Figure 2 Transmission electron micrographs of control $(A)$ and BFT exposed $(B, C)$ colonic biopsy specimens cultured for four hours. In micrographs of BFT exposed biopsy specimens there is notable vacuolation (B). Apoptotic epithelial cells are also seen (arrowed). Bar $=1 \mu \mathrm{m}$.

purified BFT into ligated intestinal loops of animals has been shown to induce acute inflammation. ${ }^{12}$

In vitro studies using confluent monolayers of the carcinoma derived HT29 colonic epithelial cell line have shown that BFT disrupts barrier function. ${ }^{30} \mathrm{BFT}$ induced separation and rounding of HT29 cells has also been reported to be associated with augmented internalisation of selected strains of enteric bacteria via the exposed lateral membranes. ${ }^{18}$ Such effects of BFT in vivo would expose the underlying lamina propria lymphocytes and macrophages to luminal bacteria and their products via pores in the basement membrane, ${ }^{31}$ with the potential for further exacerbation of mucosal inflammation.

It is of interest that in our organ culture studies, there was heterogeneity in responses to BFT by colonic epithelial cells from different individuals. On the one hand, no changes to colonocytes were seen in biopsy specimens from one individual, despite exposure to BFT for 18 hours. In contrast, severe cytotoxicity was seen in the surface and crypt epithelial cells of colonic mucosal samples from many individuals, within four hours of exposure to BFT. In two subjects, epithelial cell cytotoxicity was not observed initially but occurred at later time points (four and 18 hours).

Separate biopsy specimens from the same region of the sigmoid colon were used for test and control organ cultures, over different time periods. It is possible therefore that our results also reflect heterogeneity in responses to BFT within biopsy specimens from the same individual. However, we believe that this is highly unlikely as a cytotoxic response in the epithelial cells (to BFT) always occurred at later time points if one was seen at an early time point (subjects $2,3,4,5,6,10,11$, and 12 , in table $1)$.

Reasons for the heterogeneity between individuals in responses by colonic epithelial cells are likely to include the amount of toxin reaching the surface of epithelial cells and the subsequent outcome of the BFT-host cell interaction. Similarly, the outcome of host-BFT interactions in the colon in vivo could determine the occurrence of colonic disease in some individuals but not others. The concentration of BFT reaching the colonocytes would depend on its production by ETBF and the extent to which components of the surface mucus layer are able to inhibit its penetration to the surface of the epithelial cells. Such components of host defence include mucin glycoprotein and secretory IgA antibodies. ${ }^{32}$

In addition to the toxin induced morphological changes outlined above, there may be other similarities between ETBF and toxigenic $C$ difficile. Unresponsiveness to high concentrations of $C$ difficile toxin A by primary human colonic epithelial cells has been reported. ${ }^{20}$ Furthermore, a significant proportion of hospitalised adults are asymptomatic carriers of toxigenic $C$ difficile. ${ }^{33} 34$

Further studies should allow characterisation of the role of ETBF in the induction of diarrhoea and colonic disease in adults. Such studies include investigation of stool samples of healthy adults and those with diarrhoea for the presence of ETBF and BFT. We postulate that in susceptible individuals, diarrhoea due to ETBF would be associated with BFT induced inflammation. Histological examination of colonic biopsy specimens of patients with diarrhoea, in whom free BFT is detected in the stool sample, should allow this postulate to be tested. 
This study was supported by the Medical Research Council. The electron micrograph studies used equipment funded by the Wellcome Trust and we thank Mr Trevor Gray for assistance. D Sanfilippo was on leave of absence from Istituto di Microbio-
logia, Parma, Italy, and was supported by the University of logia, Parma, Italy, and was supported by the
Parma and CNR (contract no. 96.03300 .CT04).

1 Moore WEC, Holdeman LV. Human flora: the normal flora of twenty Japanese Hawaiians. Appl Microbiol 1974;27:961 79.

2 Moore WEC, Cato EP, Holdeman LV. Some current concepts in intestinal bacteriology. Am $\mathcal{F}$ Clin Nutr 1978;31:S33-42.

3 Myers LL, Firehammer BD, Shoop DS, et al. Bacteroides fragilis: a possible cause of acute diarrheal disease in newborn lambs. Infect Immun 1984;44:241-4.

4 Myers LL, Shoop DS, Firehammer BD, et al. Association of enterotoxigenic Bacteroides fragilis with diarrhea disease in calves. F Infect Dis 1985;152:344-7.

5 Myers LL, Shoop DS. Association of enterotoxigenic Bacteroides fragilis with diarrhea disease in young pigs. $A m$ f Vet Res 1987;48:774-5.

6 Myers LL, Shoop DS, Byars TD. Diarrhea associated with enterotoxigenic Bacteroides fragilis in foals. Am $\mathcal{F}$ Vet Res 1987;48:1565-7.

7 Weikel CS, Grieco FD, Reuben J, et al. Human colonic epithelial cells, HT-29, treated with crude Bacteroides fragilis enterotoxin dramatically alter their morphology. Infect Immun 1992;60:321-7.

8 Pantosti A, Cerquetti M, Colangeli R, et al. Detection of intestinal and extra-intestinal strains of enterotoxigenic Bacteroides fragilis by the HT-29 cytotoxicity assay. $\mathcal{F} \mathrm{Med}$ Microbiol 1994;41:191-6.

9 Mundy LM, Sears CL. Detection of toxin production by Bacteroides fragilis: assay development and screening of
extra-intestinal clinical isolates. Clin Infect Dis 1996;23: 269-76.

10 Van Tassel RL, Lyerly DM, Wilkins TD. Purification and characterization of an enterotoxin from Bacteroides fragilis. Infect Immun 1992;60:1343-50.

11 Moncrief JS, Obiso R, Barroso LA, et al. The enterotoxin of Bacteroides fragilis is a metalloprotease. Infect Immun 1995;63:175-81.

12 Obiso RJ, Lyerly DM, Van Tassel RL, et al. Proteolytic activity of the Bacteroides fragilis enterotoxin causes secre-
tion and intestinal damage in vivo. Infect Immun 1995;63: tion and

13 Sack RB, Myers LL, Hill JA, et al. Enterotoxigenic Bacteroides fragilis: epidemiologic studies of its role as a human diarrhoeal pathogens. F Diarrhoeal Dis Res 1992;10 4-9.

14 Sack RB, Albert MJ, Alam K, et al. Isolation of enterotoxigenic Bacteroides fragilis from Bangladeshi children with diarrhea: a controlled study. F Clin Microbiol 1994;32:960-3.

15 San Joakin VH, Griffis JC, Lee C, et al. Association of Bacteroides fragilis with childhood diarrhea. Scand F Infect Dis 1995;27:211-15.

16 Myers LL, Shoop DS, Stackhouse LL, et al. Isolation of enterotoxigenic Bacteroides fragilis from humans with diarrhea. F Clin Microbiol 1987;25:2330-3.
17 Pantosti A, Menozzi MG, Frate A, et al. Detection of enterotoxigenic Bacteroides fragilis and its toxin in stool sample from adults and children in Italy. Clin Infect Dis 1997;24:12-16

18 Wells CL, Van De Westerlo EMA, Jechorek RP, et al. Bacteroides fragilis enterotoxin modulates epithelial permeability and bacterial internalization by HT-29 enterocytes. Gastroenterology 1996;110:1429-37.

19 Van Tassel RL, Lyerly DM, Wilkins TD. Production of antisera against the enterotoxin of Bacteroides fragilis and their use in a cytotoxicity neutralization assay of HT-29 cells. Clin Diagn Lab Immun 1994;1:473-6.

20 Mahida YR, Makh S, Gray T, et al. Effect of Clostridium difficile toxin A on human intestinal epithelial cells: induction of interleukin 8 production and apoptosis after cell detachment. Gut 1996;38:337-47.

21 Robinson G, Gray T. Electron microscopy 2. Tissue preparation, sectioning and staining. In: Bancroft JD, Stevens A, eds. Theory and practice of histological techniques. 3rd edn. Lods. Theory and practice of histological techmiques.

22 Collins JE, Bergeland ME, Myers LL, et al. Exfoliating colitis associated with enterotoxigenic Bacteroides fragilis in a piglet. F Vet Diagn Invest 1989;1:349-51.

23 Myers LL, Collins JE, Shoop DS. Ultrastructural lesions of enterotoxigenic Bacteroides fragilis in rabbits. Vet Pathol 1991;28:336-8.

24 Myers LL, Shoop DS, Collins JE, et al. Diarrheal disease caused by enterotoxigenic Bacteroides fragilis in infant rabbits. F Clin Microbiol 1989;27:2025-30.

25 Myers LL, Shoop DS, Collins JE. Rabbit model to evaluate enterovirulence of Bacteroides fragilis. 7 Clin Microbiol 990;28:1658-60.

26 Borriello SP, Davies HA, Kamiya S, et al. Virulence factors of Clostridium difficile. Rev Infect Dis 1991;12:S185-91.

27 Branka J-E, Vallette G, Jarry A, et al. Early functional effects of Clostridium difficile toxin A on human colonocytes. Gastroenterology 1997;112:1887-94.

28 McAlindon M, Mahida YR. Cytokines and the gut. Eur 7 Gastroenterol Hepatol 1997;9:1045-50.

29 Riegler M, Sedivy T, Sogukoglu T, et al. Differential effects of Bacteroides fragilis toxin (BFT)-2 and Clostridium difficile toxin $\mathrm{A}$ and $\mathrm{B}$ on human colonic mucosa in vitro [abstract]. Gastroenterology 1997;112:A1072.

30 Obiso RJ, Azghani AO, Wilkins TD. The Bacteroides fragilis toxin fragilysin disrupts the paracellular barrier of epithelial cells. Infect Immun 1997;65:1431-9.

31 Mahida YR, Galvin A, Gray T, et al. Migration of human intestinal lamina propria lynphocytes, macrophages and eosinophils following the loss of surface epithelial cells. Clin Exp Immunol 1997;109:377-86.

32 Mahida YR. Mechanisms of host protection and inflammation in the gastrointestinal tract. $\mathcal{F} R$ Coll Physicians Lond 1997;31:493-7.

33 Gerding DN. Disease associated with Clostridium difficile infection. Ann Intern Med 1989;110:255-7.

34 Kerr RB, McLaughlin DI, Sonnenberg LW. Control of Clostridium difficile colitis outbreak by treating asymptomatic carriers with metronidazole. Am $\mathcal{F}$ Infect Control 1990;18:332-5. 